\title{
Contribution assessment of antenna structure and in-gap photocurrent in terahertz radiation of photoconductive antenna
}

Jitao Zhang, Mingguang Tuo, Min Liang, Xiong Wang, and Hao Xin

Citation: Journal of Applied Physics 124, 053107 (2018); doi: 10.1063/1.5038341

View online: https://doi.org/10.1063/1.5038341

View Table of Contents: http://aip.scitation.org/toc/jap/124/5

Published by the American Institute of Physics

\section{Articles you may be interested in}

Enhanced optical-to-THz conversion efficiency of photoconductive antenna using dielectric nano-layer encapsulation

APL Photonics 3, 051706 (2018); 10.1063/1.5021023

Half-cycle terahertz surface waves with $\mathrm{MV} / \mathrm{cm}$ field strengths generated on metal wires

Applied Physics Letters 113, 051101 (2018); 10.1063/1.5031873

Enhanced light trapping in Ge-on-Si-on-insulator photodetector by guided mode resonance effect Journal of Applied Physics 124, 053101 (2018); 10.1063/1.5031453

Deep subwavelength imaging via tunable terahertz plasmons

Applied Physics Letters 113, 051106 (2018); 10.1063/1.5035312

Perspective: Terahertz science and technology

Journal of Applied Physics 122, 230901 (2017); 10.1063/1.5007683

3D direct writing of terahertz metamaterials based on $\mathrm{TbFeO}_{3}$ dielectric ceramics

Applied Physics Letters 113, 081901 (2018); 10.1063/1.5035123

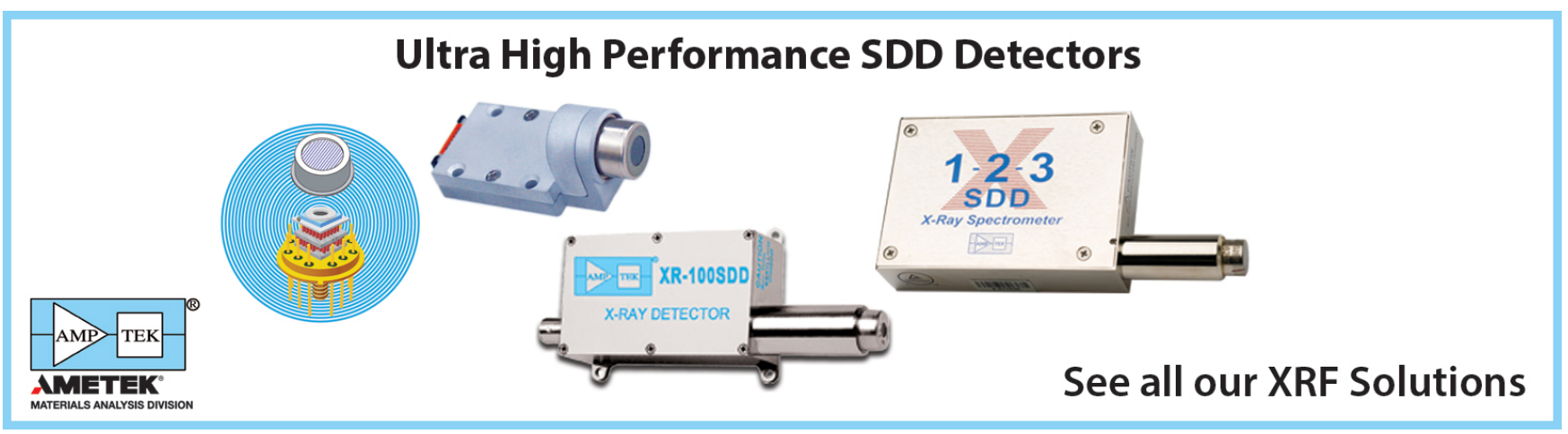




\title{
Contribution assessment of antenna structure and in-gap photocurrent in terahertz radiation of photoconductive antenna
}

\author{
Jitao Zhang, ${ }^{1,2}$ Mingguang Tuo, ${ }^{1}$ Min Liang, ${ }^{1}$ Xiong Wang, ${ }^{1,3}$ and Hao Xin ${ }^{1}$ \\ ${ }^{1}$ Department of Electrical and Computer Engineering, University of Arizona, Tucson, Arizona 85721, USA \\ ${ }^{2}$ Fischell Department of Bioengineering, University of Maryland, College Park, Maryland 20742, USA \\ ${ }^{3}$ School of Information Science and Technology, ShanghaiTech University, Shanghai 201210, China
}

(Received 2 May 2018; accepted 18 July 2018; published online 7 August 2018)

\begin{abstract}
Photoconductive antenna (PCA) is one of the most widely used terahertz (THz) devices nowadays. Although PCAs have been extensively studied through both theoretical analysis and device design, there still lacks a common agreement upon the mechanism of $\mathrm{THz}$ radiation. One of the central questions is how to distinguish and assess the contribution of the antenna structure and in-gap photocurrent to the overall radiation of a PCA. In this work, a three-dimensional full-wave model was first used to quantify the overall far-field radiation of PCAs. The commercial solver (i.e., HFSS) and the Hertzian dipole approximation method were then applied to quantify the far-field radiation solely from the antenna structure and in-gap photocurrent, respectively. The contribution of the antenna structure and in-gap photocurrent can therefore be distinguished by comparing the simulation results among the three methods. The results suggest that, although the $\mathrm{THz}$ radiation originates from laser-excited photocurrent within the gap, the overall $\mathrm{THz}$ radiation of a PCA is predominated by the antenna structure. As a validation, the cancellation effect was predicated by numerical simulation of coplanar stripline PCA and confirmed with experiment using butterfly shaped stripline PCA. The presented work uncovers the details of the underlying radiation mechanism of the PCA. This could inspire PCA design that aims for engineering the radiation properties of a PCA for specific applications. Published by AIP Publishing. https://doi.org/10.1063/1.5038341
\end{abstract}

\section{INTRODUCTION}

Terahertz (THz) photoconductive antenna (PCA) has been extensively used in applications spreading over various fields, such as non-destructive inspection, biomedical diagnosis, security screening, and chemical fingerprinting. ${ }^{1-3}$ The earliest demonstration of $\mathrm{THz}$ radiation from a PCA was accomplished by Auston et al. Thus, it is also known as the Auston switch, ${ }^{4,5}$ which was fabricated by depositing dipolelike electrodes on an epitaxial silicon film on a sapphire substrate. In this pioneer work, $\mathrm{THz}$ radiation of this PCA device was attributed to the decay of the in-gap photocurrent excited by an ultrafast laser pulse. In addition, the geometry of the electrodes was found to influence the response of the $\mathrm{THz}$ radiation but it was not fully investigated. ${ }^{6,7}$ Since then, massive effort has been devoted to understanding and manipulating the radiation properties of PCAs by means of both theoretical/numerical analysis ${ }^{7-18}$ and experiments, ${ }^{6,10,11,19-27}$ which greatly extend our understandings of the $\mathrm{THz}$ radiation mechanism therein. Recently, nanostructures were integrated into the structural design of PCAs and significant enhancements in THz radiation have been observed. ${ }^{28-31}$ In addition, the combination of vertically illuminated PCA and unitraveling carrier design has shown promising enhancement of $\mathrm{THz}$ radiation. ${ }^{32}$ Regardless of those great achievements, there still lacks a universal physical explanation of the $\mathrm{THz}$ radiation mechanism of the PCA. One of the open questions is how to distinguish and assess the contribution of the antenna structure and in-gap photocurrent in the radiation process. The answer to this question is desired for engineering the radiation properties of PCAs.
Currently, the physical models that have been developed to describe the far-field radiation process of a PCA can be categorized into three types. The first type of model attributes the $\mathrm{THz}$ radiation to the laser-excited photocurrent $J$ within the gap region in between the electrodes. Since the transient in-gap photocurrent oscillates in picoseconds, it will excite THz-band radiation in the far field, which is proportional to the time derivative of the photocurrent via $E \propto d J / d t$ (Hertzian dipole approximation method). In this context, the PCA was treated as an electrically small point dipole since the dimension of the antenna structure is usually much smaller than the wavelength of the radiation, and the contribution of the antenna structure was not within consideration. The photocurrent was then deduced from the carrier dynamics by using the current surge model ${ }^{33,34}$ or the DrudeLorentz model. ${ }^{8,9}$ However, many experiments show that the $\mathrm{THz}$ radiation (especially the bandwidth) of a PCA is strongly related to the dimension/geometry of the antenna structure. ${ }^{5,21,23}$ To address this issue, a second type of model was developed with the hypothesis that the $\mathrm{THz}$ radiation of the PCA is solely from the antenna structure, which works as a common electric antenna fed by a photocurrent source. A representative is the equivalent circuit model, ${ }^{12,13,35-37}$ in which the PCA is described as a lumped-element circuit. To ensure a closed circuit, this model assumes that the gap region is fully illuminated by a laser beam, which, unfortunately, is not true for many cases. ${ }^{38-40}$ In addition, the generation of photocurrent and far-field radiation is calculated independently in this model, while in reality, they happen simultaneously and are coupled together; thus, changing one 
will eventually affect the other. The representative of the third type is the three-dimensional (3D) full-wave model, in which the generation of photocurrent in all places (i.e., within the gap and underneath the antenna structure) and the electromagnetic radiation are inherently coupled together based on the physical behaviors of the photoconductive device. ${ }^{14-16,41}$ To implement the coupling in the model, the real-time photocurrent calculated from carrier dynamics equations is used as the excitation source in Maxwell's equations, and the time-varying electric fields as well as the photocurrent can then be calculated and updated at every time step by using the finite-difference time-domain (FDTD) method. ${ }^{42}$ On one hand, the full-wave model provides a comprehensive approach to predict the $\mathrm{THz}$ radiation of a PCA by incorporating the effect of both in-gap photocurrent and antenna structure. On the other hand, however, it is difficult to distinguish and quantify the contribution of these two mechanisms to the far-field $\mathrm{THz}$ radiation because of the inherent coupling, which hinders our understanding of the radiation mechanism of the PCA.

In this work, the contribution from different regions (i.e., antenna structure and in-gap photocurrent) to the radiation of a PCA was distinguished and quantified through numerical simulation. The 3D full-wave model was first implemented to simulate the overall $\mathrm{THz}$ radiation of a PCA. The calculated photocurrent on the electrode was then extracted from full-wave model simulation and treated as a feeding source for the antenna structure, and the $\mathrm{THz}$ radiation from the antenna structure was simulated by the commercial solver (i.e., HFSS). In addition, the THz radiation from in-gap photocurrent was estimated by using the Hertzian dipole approximation method $(E \propto d J / d t)$. The contribution of each factor to the far-field $\mathrm{THz}$ radiation of the PCA can thus be quantified by comparing the results with those from the full-wave model. As a demonstration of this methodology and without loss of generality, two typical PCAs (i.e., a dipole PCA and a coplanar stripline PCA) were investigated. The results indicated that, although the $\mathrm{THz}$ radiation originates from laser-excited photocurrent within the gap, the far-field $\mathrm{THz}$ radiation of a PCA is dominated by the antenna structure. Furthermore, as a proof-of-concept validation, the cancellation effect of the symmetric coplanar stripline PCA was predicated by numerical simulation and verified in experiment.

\section{PRINCIPLE AND METHODS}

The far-field radiation of a PCA originates from the optics-to-THz conversion within the device. When an ultrafast femtosecond $(f s)$ laser pulse illuminates the photoconductive material within the gap region in between the electrodes, free electron-hole pairs are generated. Due to the establishment of a potential field across the gap by externally biasing the electrodes, the generated electrons and holes move to the anode and cathode, respectively, inducing a transient photocurrent in the gap at the picosecond (ps) time scale, which therefore results in far-field $\mathrm{THz}$ radiation according to Maxwell's equations. In addition, if the generated carriers are sufficiently close to the electrodes so that the carriers can migrate to the electrode within its lifetime,$^{29,43}$ the photocurrent acts as a driving source of the antenna structure, which therefore radiates $\mathrm{THz}$ radiation as well. However, the radiation from both mechanisms cannot be readily distinguished.

The model developed here is based on two full-wave analyses that were previously proposed by Sano and Shibata and Moreno et al. ${ }^{14,16}$ In this model, the carrier dynamics equations (including continuity equations and drift-diffusion equations) were coupled with Maxwell's equations. To achieve this, the photo-excited currents calculated by the carrier dynamics equations were used as the excitation source of Maxwell's equations, and the time-varying electric fields as well as the photo-excited currents at each time step were then calculated. The detailed implementation of the fullwave model is described in Appendix.

Equation (A2) indicates that all of the current sources (both in the gap and the region underneath the antenna structure) within the calculation volume were considered as the driving current of radiation in the full-wave model. The radiation pattern also took into consideration the antenna structure in the approximation of a perfect electric conductor (PEC). Therefore, the simulated results represent the overall contribution of both mechanisms. To distinguish the contribution of each, the calculated photocurrent was first extracted from the simulation of the full-wave model. The radiation from the antenna structure was then evaluated by using transient photocurrent (the total current underneath the antenna structure) as the drive source in HFSS simulation, while the radiation from in-gap photocurrent was estimated via the Hertzian dipole approximation method. By comparing the corresponding simulation result with the far-field $\mathrm{THz}$ radiation calculated from the full-wave model, the contribution of the antenna structure and in-gap photocurrent can be distinguished.

Without loss of generality, two representative PCAs (i.e., dipole and coplanar stripline) were used in the simulation. The geometries are shown in Fig. 1. The dipole PCA had a small gap size of $5 \mu \mathrm{m}$, which was illuminated by the same-size laser spot. The dipole length (in the $x$-direction) was varied to study its impact on the far-field $\mathrm{THz}$ radiation. The coplanar stripline PCA had a large gap size of $34 \mu \mathrm{m}$, which was anode-illuminated by a laser spot of $20-\mu \mathrm{m}$ diameter for optimized $\mathrm{THz}$ radiation. The photoconductive material used in the model was low-temperature-grown gallium

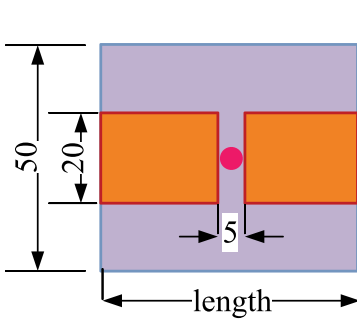

(a) Dipole PCA

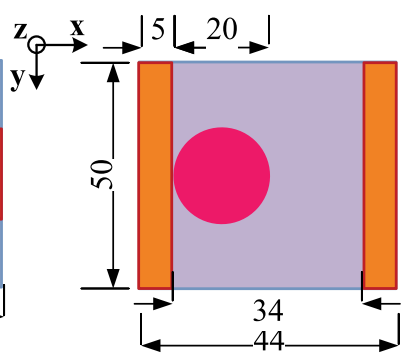

(b) Stripline PCA
FIG. 1. Geometry of (a) dipole PCA and (b) coplanar stripline PCA. The thickness of the metallic layer is $0.2 \mu \mathrm{m}$, and the thickness of the substrate (in $z$-direction) for both PCAs used in the later implemented full-wave FDTD model is $2 \mu \mathrm{m}$. All of the numbers are in units of micrometer. 
arsenide (LT-GaAs), whose electron and hole carrier lifetimes are $0.1 \mathrm{ps}$ and $0.4 \mathrm{ps},{ }^{44}$ and the initial electron and hole mobilities are $200 \mathrm{~cm}^{2} / \mathrm{Vs}$ and $30 \mathrm{~cm}^{2} / \mathrm{V} \mathrm{s}{ }^{45,46}$ The saturated velocity of the electron and hole is $1 \times 10^{7} \mathrm{~cm} / \mathrm{s}^{47}$ The relative permittivity of the material is 12.9 , and the absorption coefficient is $1 \times 10^{4} \mathrm{~cm}^{-1}$. ${ }^{48}$ The intrinsic carrier concentration is $2.1 \times 10^{6} \mathrm{~cm}^{-3}$, and the n-type doping concentration of the impurities $N_{D}-N_{A}$ is $1.5 \times 10^{17} \mathrm{~cm}^{-3}$. 49 The exciting laser pulse had a wavelength of $800 \mathrm{~nm}$, a pulse-duration of $80 \mathrm{fs}$, and a repetition rate of $80 \mathrm{MHz}$. The averaged laser power was $2.6 \mathrm{~mW}$, thus the peak intensity $I_{0}$ would vary with the size of the beam spot. The simulation was run for a total time period of 2 ps with a step of $0.33 \mathrm{fs}$, and the temporal peak $t_{0}$ of the laser pulse was set at $0.5 \mathrm{ps}$. The DC bias voltage of the PCA was $5 \mathrm{~V}$. The interface between the metallic structure and the semiconductor was set as Ohmic contact, and the PEC was used as the boundary condition for the electrodes. The far-field $\mathrm{THz}$ radiation was calculated at the point 200-mm away from the PCA along the $z$-direction and with the coordinate of $(0,0$, and $-200000 \mu \mathrm{m})$.

\section{RESULTS AND DISCUSSION}

\section{A. Dipole PCA}

The far-field radiation of dipole PCAs with different lengths (i.e., $30 \mu \mathrm{m}, 60 \mu \mathrm{m}, 90 \mu \mathrm{m}, 120 \mu \mathrm{m}$, and $200 \mu \mathrm{m}$ ) was simulated by the full-wave model. The calculated transient photocurrent at the vicinity of the anode electrode was used as the excitation source in the HFSS transient solver to estimate the far-field $\mathrm{THz}$ radiation solely from the antenna structure. As a representative example, Fig. 2 shows the distribution of the transient current density on the top surface of the dipole PCA with $60-\mu \mathrm{m}$ dipole length at $0.5 \mathrm{ps}$. The simulation results of the HFSS simulation were compared with those of the full-wave model, as shown in Fig. 3.

For all cases, the results (absolute values) obtained by the full-wave model and the HFSS simulation agree well with each other. Since the simulation of HFSS only involved the current that flows on the antenna structure, it suggests that the radiation of the dipole PCA is predominant by the antenna structure. In addition, the results show that the

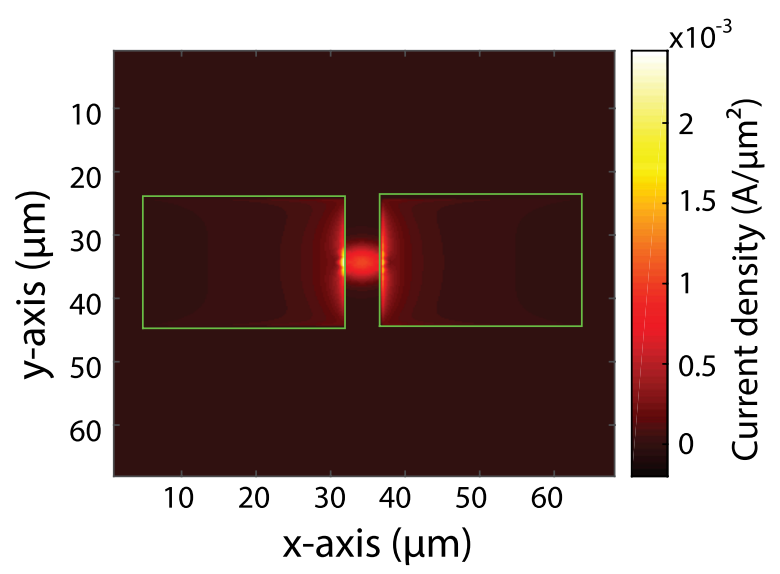

FIG. 2. Distribution of transient photocurrent density on the top surface of the dipole PCA at 0.5 ps. The color bar has the unit of $\mathrm{A} / \mu \mathrm{m}^{2}$. The green solid line indicates the electrodes of the PCA.
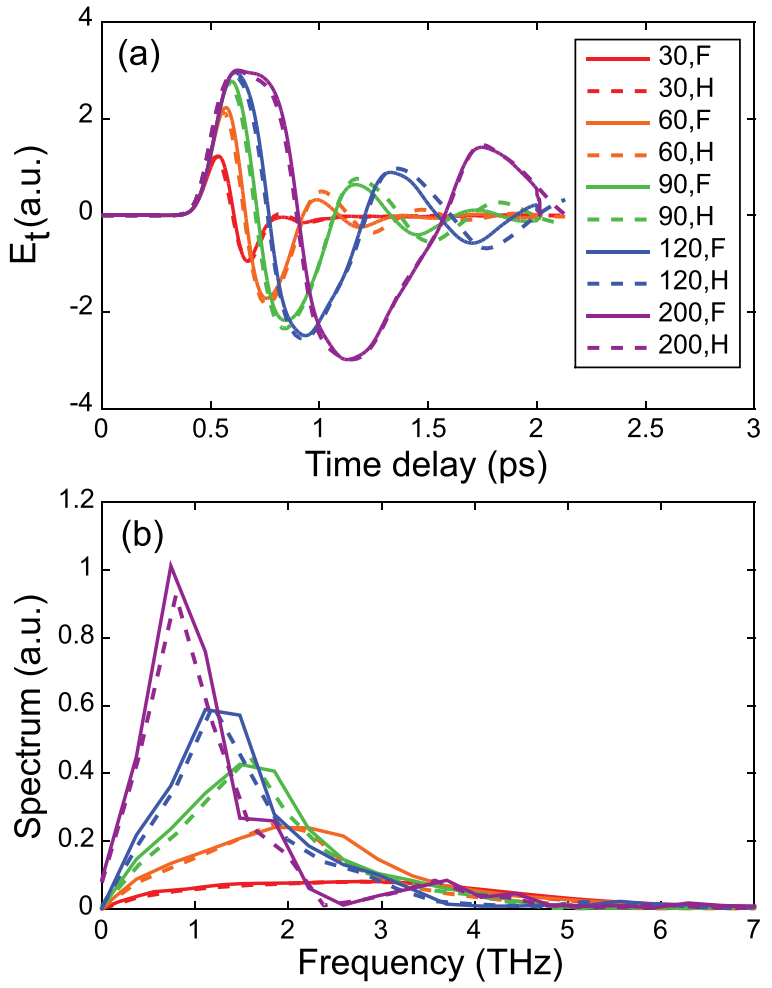

FIG. 3. Simulation results of the far-field $\mathrm{THz}$ radiation of the dipole PCAs with different lengths. (a) Time-domain E-field, which is parallel to the current flowing direction (in the $x$-direction in Fig. 1), and (b) corresponding spectra calculated by fast-Fourier-transform (FFT). The numbers in the legend indicate the length of the dipole in units of micrometer, and " $\mathrm{F}$ " and " $\mathrm{H}$ " indicate the results obtained by the full-wave model and HFSS simulation.

spectral bandwidth strongly depends on the length of the dipole, i.e., increasing of the dipole length results in the redshift of the peak frequency, consistent to experimental observation. ${ }^{23}$ Moreover, it is found that the amplitude of the peak frequency increases with the increase in the dipole's length. This can be understood by the mismatch between the timevarying source impedance and the antenna impedance. In practice, the peak frequency of the dipole PCA is usually not at the antenna's resonance but at the frequency where the mismatch is at minimum. ${ }^{50}$

The far-field $\mathrm{THz}$ radiation was also estimated using the Hertzian dipole approximation $(E \propto d J / d t)$, where $J$ was the photocurrent within the gap center and extracted from the full-wave simulation. Figure 4 shows the photocurrents and corresponding far-field $\mathrm{THz}$ radiation fields, which show that the temporal shapes of the photo-excited currents changed very little with regard to the dipole length of PCAs. More importantly, the waveform of estimated E-fields by Hertzian dipole approximation [Fig. 4(b)] distinctly differed from the results of the full-wave model [Fig. 3(a)].

Figure 5 shows the comparison between the full-wave model and Hertzian dipole approximation for dipoles with lengths of $30 \mu \mathrm{m}$ and $200 \mu \mathrm{m}$. The time-domain pulse signals are normalized to their peaks for convenience. Figures 5(a) and 5(c) suggest that, for the 30- $\mu$ m-long dipole PCA, the estimated $\mathrm{THz}$ radiation from Hertzian dipole approximation and that from the full-wave model share similar features. This is because the length of the dipole structure is sufficiently 

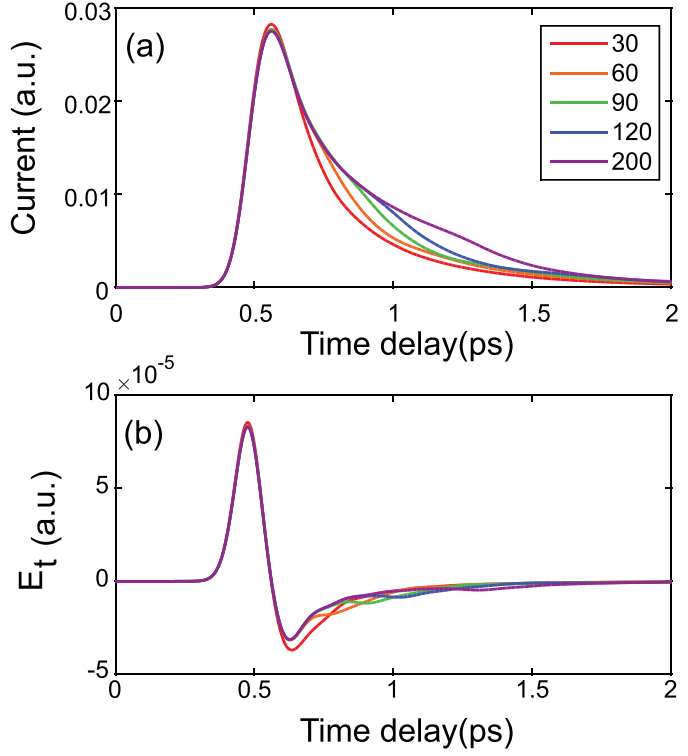

FIG. 4. Simulated (a) photo-excited current and (b) corresponding far-field $\mathrm{THz}$ radiated field using the Hertzian dipole approximation for dipole PCAs. The numbers in the legend represent the length of the dipole PCAs in units of micrometer.

short so that it can be approximated as an electrically small point source. However, for the 200- $\mu$ m-long dipole PCA, a large discrepancy can be observed between those two methods, as shown in Figs. 5(b) and 5(d). It suggests that the estimation of the Hertzian dipole approximation is inadequate for long dipole PCA, and the contribution of the antenna structure has to be taken into consideration in this context.

\section{B. Coplanar stripline PCA}

The antenna structure of a coplanar stripline PCA consists of a pair of parallel lines, as shown in Fig. 1(b). Since
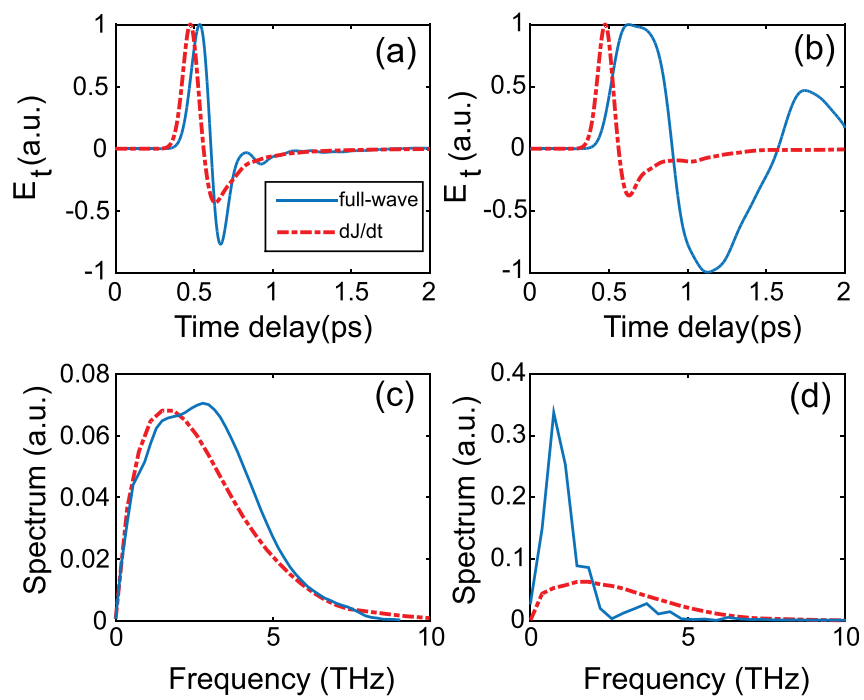

FIG. 5. Comparison of the far-field $\mathrm{THz}$ radiation calculated by different methods (solid blue line: full-wave model, dash-dotted red line: Hertzian dipole approximation). (a) Radiated field of 30- $\mu$ m-long dipole PCA and (c) corresponding spectra calculated by FFT, and (b) radiated field of $200-\mu \mathrm{m}$ long dipole PCA and (d) corresponding spectra calculated by FFT. All of the time-domain signals are normalized to their peaks. the PCA is partially illuminated by a laser beam smaller than its gap size, the photo-excited current is distributed nonuniformly within the photoconductive gap, as shown in Fig. 6. In this case, the HFSS simulation cannot be implemented because a uniform current source across the PCA gap is assumed therein. Instead, one can utilize the Hertzian dipole approximation to estimate its far-field radiation. In this case, the current extracted from the center of the illuminated region was chosen as current source. The comparison of the far-field $\mathrm{THz}$ radiations deduced by the full-wave model and by the Hertzian dipole approximation is shown in Figs. 7(a) and 7(b). The shapes of the time-domain signals from both methods agree well with each other. To distinguish the contribution of the in-gap photocurrent from the antenna structure, we can move the laser beam from the anode to the cathode horizontally and monitor the change of the $\mathrm{THz}$ field. During this process, the radiation from the antenna structure will decrease because less photo-excited electrons can reach and feed the anode, while the radiation from the in-gap photocurrent will not be affected. In case the laser beam is far enough from the anode so that none of photo-excited electrons can arrive at the anode before recombination, the $\mathrm{THz}$ radiation will be solely from the in-gap photocurrent. Figure 7(c) shows the simulation results. The $\mathrm{THz}$ radiation decreases monotonically as the laser beam was moved away from the anode. At the farthest position (12 $\mu \mathrm{m}$ away from the anode), the amplitude dropped to about $18 \%$ of the highest value. A further movement will cause the blocking of the laser beam by the cathode thus not taken into consideration. We estimate that the in-gap photocurrent and the antenna structure contribute about $18 \%$ and $82 \%$ of the overall THz radiation of Fig. 7(a), respectively. This indicates that the antenna structure also dominates the $\mathrm{THz}$ radiation of coplanar stripline PCA, while the contribution of the in-gap photocurrent is much smaller but cannot be ignored.

Furthermore, from Fig. 7(a), we observed that the $\mathrm{THz}$ field calculated by the full-wave model has additional fluctuations after the main peak. To explore the underlying mechanism of the time-domain fluctuations in Fig. 7(a), the

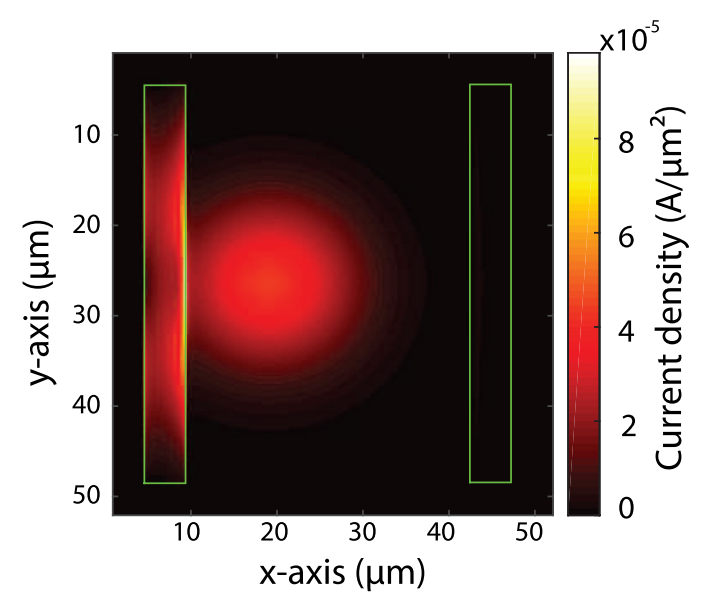

FIG. 6. Distribution of the transient current density on the top surface of the coplanar stripline PCA at $0.5 \mathrm{ps}$. The color bar has the unit of $\mathrm{A} / \mu \mathrm{m}^{2}$. The green solid line indicates the electrodes of the PCA. 

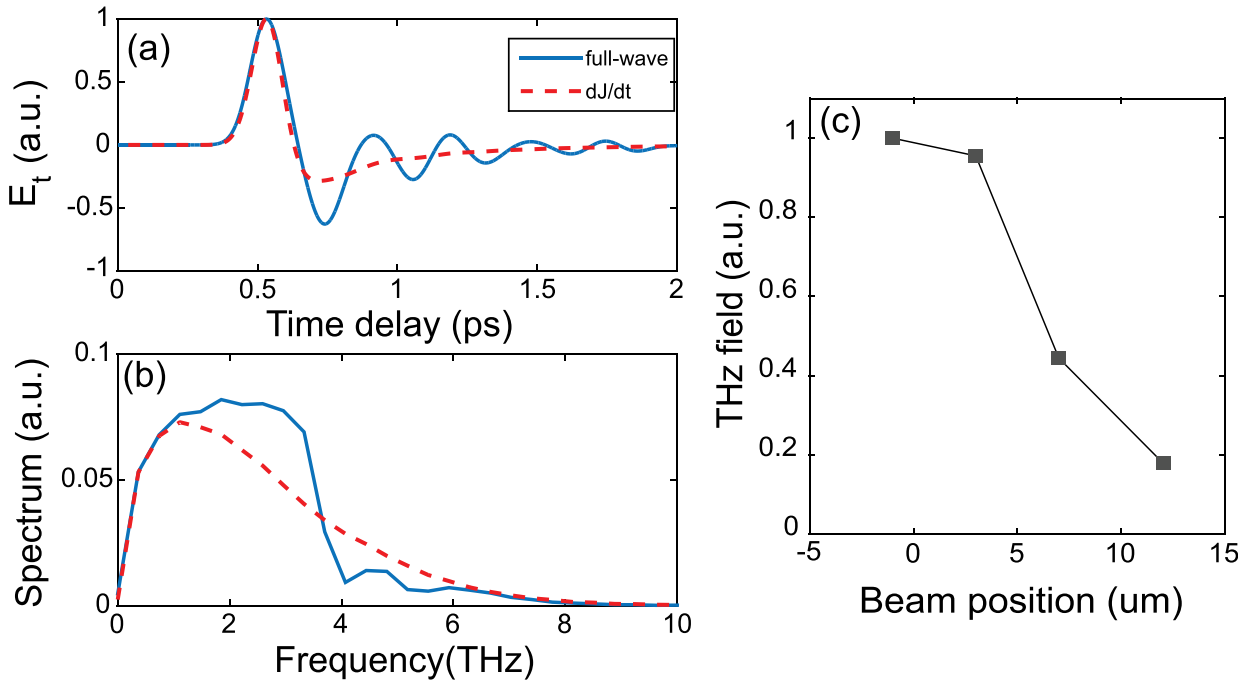

FIG. 7. Simulation result of the farfield $\mathrm{THz}$ radiated field of the coplanar stripline PCA by the full-wave model and the Hertzian dipole approximation. (a) Normalized time-domain radiated fields and (b) corresponding spectra calculated by FFT. (c) Peak of the THz field corresponding to the location of the beam spot; the number of $\mathrm{x}$-axis indicates the distance from the inside edge of the anode to the left edge of the beam spot. Negative distance means the beam edge is slightly blocked by the anode. length of the stripline (along the $y$-axis) was varied and the corresponding far-field $\mathrm{THz}$ radiations were simulated by the full-wave model (Fig. 8), which were further compared with those calculated by the Hertzian dipole approximation (Fig. 9). In Fig. 8, the main peaks of the time-domain $\mathrm{THz}$ fields overlap well with each other for all cases, and the fluctuations are delayed with regard to the length of the stripline, suggesting the fluctuations are due to the reflection of current by the ends of the stripline. When the length of the stripline is comparable to the size of the beam spot (for example, $25-\mu \mathrm{m}$ case), the fluctuations disappear. However, no fluctuation was ever observed for Hertzian dipole approximation in Fig. 9. By comparing Fig. 8 with Fig. 9, it indicates that for the stripline PCA, both in-gap photocurrent and antenna structure contribute to the overall far-field $\mathrm{THz}$ radiation. In particular, from the time-domain signals of Figs. 8(a) and 9(a), it can be further concluded that the
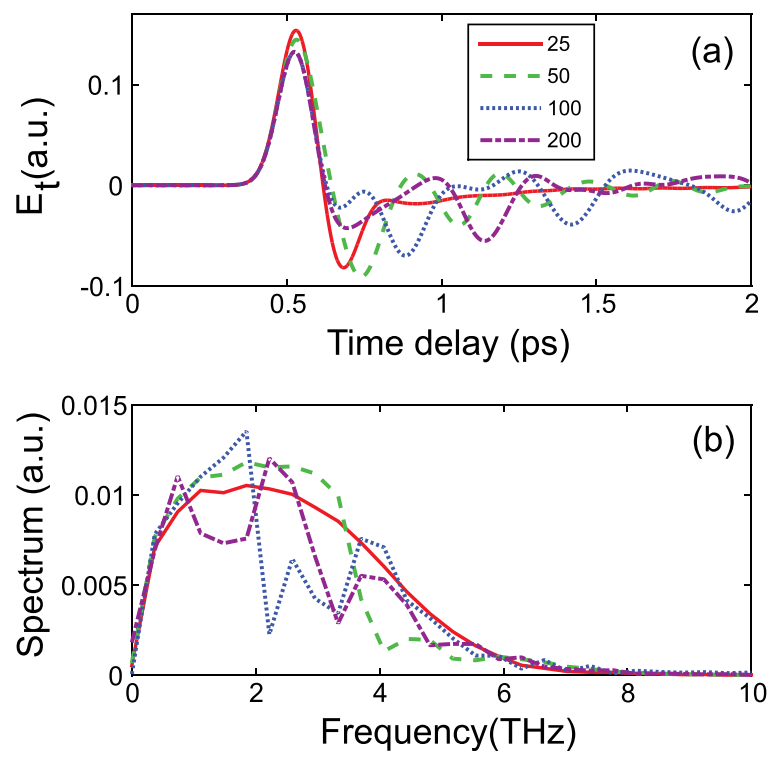

FIG. 8. Simulated far-field THz radiations of the stripline PCAs with different lengths by the full-wave model. (a) Time-domain radiated fields and (b) corresponding spectra calculated by FFT. The numbers in the legend represent the lengths of the stripline PCAs in units of micrometer. fluctuations after the leftmost main peak are due to the reflected current on the antenna structure.

\section{Cancellation effect}

In Sec. III B, the laser beam spot was located in the middle of the stripline along the $y$-direction; thus the photoexcited current was distributed symmetrically on the electrode. In this case, one can expect that the radiation along the $y$-direction will be cancelled out due to the existence of this symmetry. It is thus interesting to investigate what will happen when the symmetry is broken. For this purpose, identical stripline PCA in Fig. 1(b) was simulated by the full-wave model. The length of the stripline was $50 \mu \mathrm{m}$, and the laser power was $6 \mathrm{~mW}$. The rest of the parameters were kept the same. For the symmetric illumination case [Fig. 10(a)], the beam spot was located in the middle along the strip. For the asymmetric case [Fig. 10(b)], the laser beam spot was moved
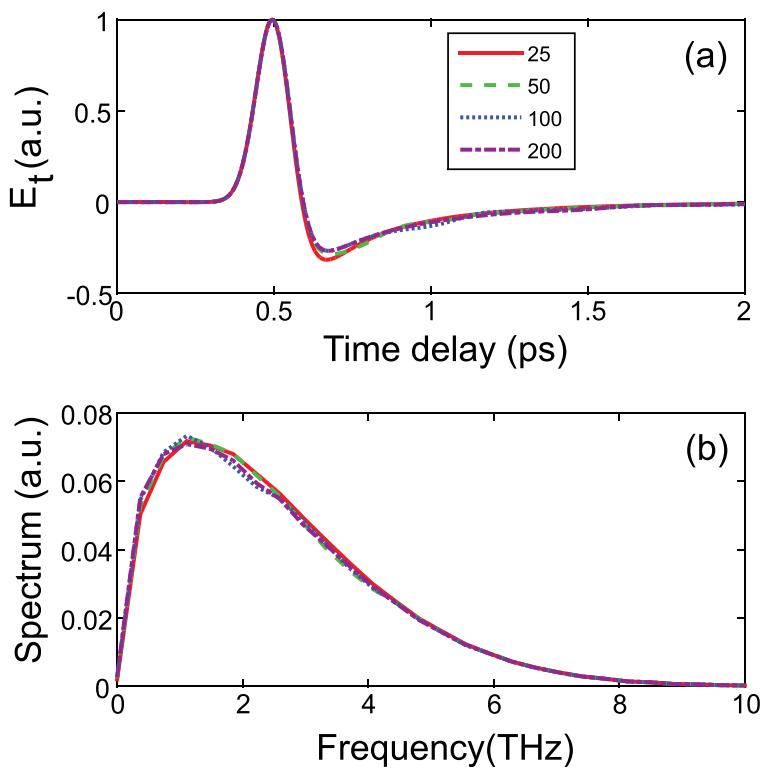

FIG. 9. Simulated far-field THz radiation of the stripline PCAs with different lengths by Hertzian dipole approximation. (a) Time-domain radiated fields and (b) corresponding spectra calculated by FFT. The numbers in the legend represent the lengths of the stripline PCAs in units of micrometer. 

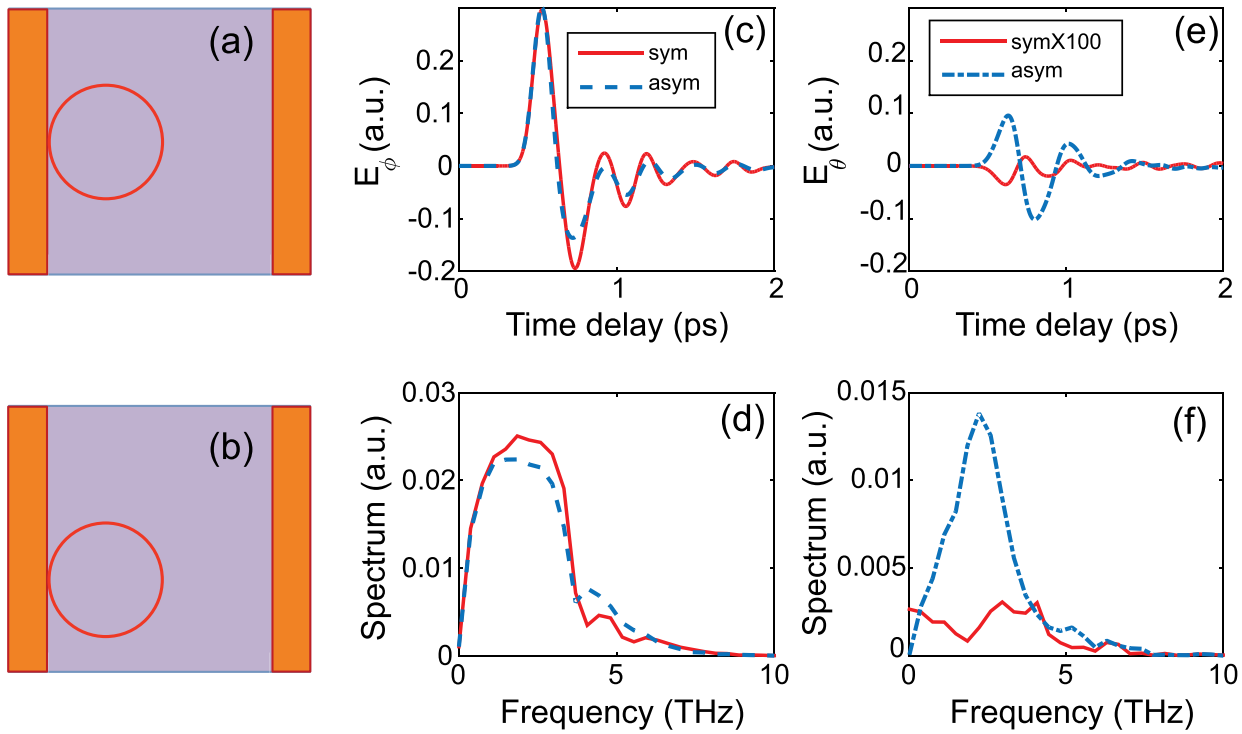

FIG. 10. Simulated far-field THz radiations of the stripline PCAs with symmetric and asymmetric illumination. (a) Symmetric illumination and (b) asymmetric illumination. (c) Time-domain field in the $x$-direction and (d) corresponding spectra. (e) Time-domain field in the $y$-direction and (f) corresponding spectra. The curve of the symmetric illumination case is magnified by 100 times as labeled. The "sym" and "asym" in the legend represent the symmetric and asymmetric illumination, respectively.
$10 \mu \mathrm{m}$ away from the middle along the $y$-direction. The farfield $\mathrm{THz}$ radiations along the $x$ - and $y$-directions (i.e., $E_{\phi}$ and $E_{\theta}$ ) were calculated, and the results are presented in Fig. 10. In the symmetric case, the dominate radiation field is in the $x$-direction $\left(E_{\phi}\right)$, which is 100 times stronger than that in the $y$-direction $\left(E_{\theta}\right)$. However, with asymmetrical illumination, the radiation field in the $y$-direction $\left(E_{\theta}\right)$ is hundred times enhanced and comparable (i.e., 1/3) to $E_{\phi}$. It therefore indicates that the radiation in the $y$-direction can be significantly enhanced by breaking the symmetry, by which the cancellation effect was removed.

A proof-of-concept experiment was designed to verify this cancellation effect. A butterfly shaped stripline PCA was used in the experiment and its dimension is shown in Fig. 11(a). The central region of this PCA was a stripline with a length of $100 \mu \mathrm{m}$, a gap size of $34 \mu \mathrm{m}$, and a line width of $5 \mu \mathrm{m}$. The ends of the stripline were gradually extended to four bigger pads which were named as "wings." Because of the considerably large dimension of the wings, this PCA had a relatively good performance at the sub-THz regime. Owing to the butterfly shape, each wing generates $\mathrm{THz}$ radiation components in both horizontal and vertical directions. However, with the laser spot illuminating at the middle of the stripline, the horizontal radiation will be

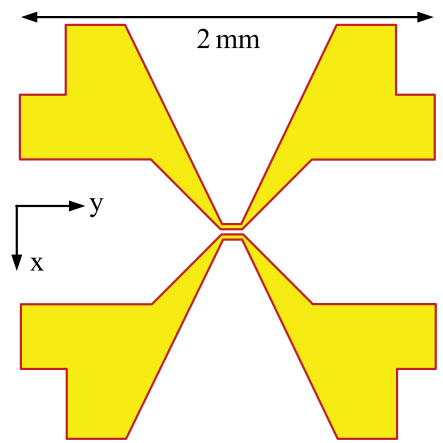

(a)

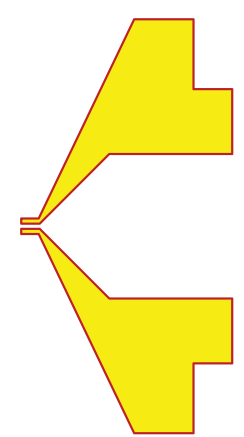

(b)
FIG. 11. Structure and dimension of (a) the whole-wing butterfly shaped stripline PCA and (b) the half-wing stripline PCA used in the experiment. cancelled out due to the symmetry. To break this symmetry, instead of moving the laser beam spot position, another antenna with only half of the wings [Fig. 11(b)] was designed.

The antenna structures were fabricated on the GaAs-onsapphire wafer by optical lithography and lift-off techniques. $^{51}$ The metallic layer was made of $40 \mathrm{~nm} \mathrm{Cr} / 200 \mathrm{~nm}$ $\mathrm{Au}$. A passivation layer of $\mathrm{SiO}_{2}$ was deposited on the top of the gap region to improve the optical transmission and thermal properties of the device. The individual PCA chip was then attached to a hyper-hemispherical silicon lens before the test. An in-house-built $\mathrm{THz}$ time-domain spectroscopy (THz-TDS) was used to measure the far-field $\mathrm{THz}$ radiation of the PCAs, which served as the THz emitters. A commercial dipole PCA fabricated on LT-GaAs (PCA-44-06-10800-h, BATOP GmbH) was used as the THz detector. Since the dipole PCA is only sensitive to the $\mathrm{THz}$ field parallel to the dipole orientation, by aligning the dipole PCA detector horizontally and vertically, the two orthogonal radiation field components of the PCA emitter can be measured independently. In the experiment, the PCA emitter was externally biased at $5 \mathrm{~V}$ and illuminated by a $4 \mathrm{~mW}$ laser beam, which has a diameter of $20 \mu \mathrm{m}$ at the focal plane. The beam spot was close to the anode to get optimized $\mathrm{THz}$ radiation. The PCA detector was illuminated by a $5 \mathrm{~mW}$ laser beam to achieve an optimized signal-to-noise ratio. The signal of the dipole detector was collected by a lock-in-amplifier (SR850, Stanford Research Systems).

The measurement results are shown in Fig. 12, in which the time-domain data of the whole-wing structure were arbitrary delayed for the convenience of comparison. For the whole-wing structure, the time-domain peak of the radiation in the $x$-direction is 5.3 times larger than that in the $y$-direction [Figs. 12(a) and 12(b)]. For the half-wing structure, however, the ratio of the two components drops to 1.6, with the expense that the radiation in the $x$-direction drops by $25 \%$. According to Fig. 12(b), the radiation of the half-wing structure is enhanced by about 2.5 times in the $y$-direction compared to that of the whole-wing structure. Therefore, the overall enhancement is still remarkable [Figs. 12(c) and 12(d)]. The experimental results of Fig. 12 confirm the 

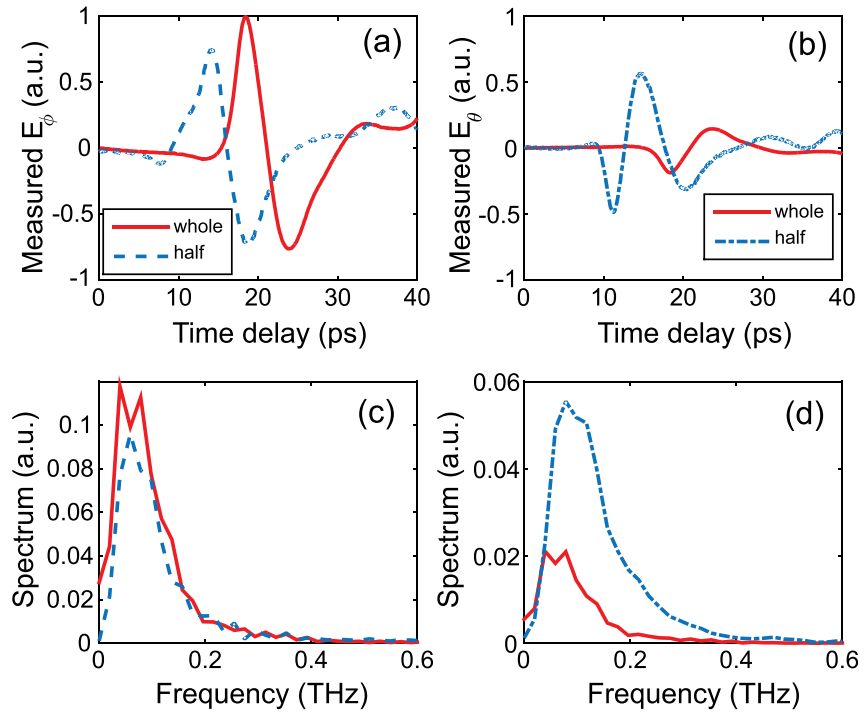

FIG. 12. Experiment results of the whole- and half-wing butterfly shaped stripline PCAs. (a) Measured far-field radiated field in the $x$-direction and (c) corresponding spectra. (b) Measured far-field radiated field in the $y$-direction and (d) corresponding spectra. The time-domain fields of the whole PCA in (a) and (b) are arbitrary delayed, and all of the time-domain data are normalized to the peak of the radiated field of the whole PCA.

existence of the cancellation effect predicted by the simulation of Fig. 10. The slight decrease in the $\mathrm{THz}$ radiation in the $x$-direction of the half-wing structure is expected. Because each wing of the butterfly shaped PCA contributes to the $\mathrm{THz}$ radiation in both directions, cutting the wings not only breaks the symmetry in the $y$-direction, but also results in less radiation in the $x$-direction.

\section{CONCLUSION}

The radiation mechanism of PCA was investigated by both numerical simulation and experiment. The contribution of the antenna-structure and in-gap photocurrent to far-field $\mathrm{THz}$ radiation of a PCA was distinguished and quantified by incorporating the 3D full-wave model with the HFSS and Hertzian dipole approximation method. The results indicate that although the overall radiation of a PCA is contributed by both mechanisms, the antenna structure always plays a predominant role. As a proof-of-concept validation, the cancellation effect of coplanar stripline PCA was predicated by numerical simulation and confirmed with the experiment. This work provides a new approach to understand the underlying radiation mechanism of the PCA. With the proposed method, the role of the antenna structure and in-gap photocurrent in the far-field $\mathrm{THz}$ radiation of a PCA can be predicted. Therefore, this work could help inspire a new PCA design that features engineered radiation properties for specific applications.

\section{ACKNOWLEDGMENTS}

This work was supported by the National Science Foundation Major Research Instrument Program under Grant No. 1126572.

\section{APPENDIX: PHYSICAL MODEL}

The involved equations of the full wave model are listed as below

$$
\begin{gathered}
\nabla \times \vec{E}(\vec{r}, t)=-\mu \frac{\partial \vec{H}(\vec{r}, t)}{\partial t}, \\
\nabla \times \vec{H}(\vec{r}, t)=\varepsilon \frac{\partial \vec{E}(\vec{r}, t)}{\partial t}+\vec{J}_{n}(\vec{r}, t)+\vec{J}_{p}(\vec{r}, t), \\
q \frac{\partial n(\vec{r}, t)}{\partial t}=\nabla \cdot \vec{J}_{n}(\vec{r}, t)+q(G(\vec{r}, t)-R(\vec{r}, t)), \\
q \frac{\partial p(\vec{r}, \mathrm{t})}{\partial t}=-\nabla \cdot \vec{J}_{p}(\vec{r}, t)+q(G(\vec{r}, t)-R(\vec{r}, t)), \\
\vec{J}_{n}(\vec{r}, t)=q \mu_{n} n(\vec{r}, t)\left(\vec{E}_{D C}(\vec{r})+\vec{E}(\vec{r}, t)\right) \\
+q \mu_{n} n_{D C}(\vec{r}) \vec{E}(\vec{r}, t)+q D_{n} \nabla n(\vec{r}, t), \\
\vec{J}_{p}(\vec{r}, t)= \\
q \mu_{p} p(\vec{r}, t)\left(\vec{E}_{D C}(\vec{r})+\vec{E}(\vec{r}, t)\right) \\
+q \mu_{p} p_{D C}(\vec{r}) \vec{E}(\vec{r}, t)-q D_{p} \nabla p(\vec{r}, t),
\end{gathered}
$$

where $\vec{E}(\vec{r}, t)$ and $\vec{H}(\vec{r}, t)$ are the time- and spatialdependent electric and magnetic fields, $\varepsilon$ and $\mu$ are the permittivity and permeability, $q$ is the elementary charge, $\vec{J}_{n}(\vec{r}, t)$ and $\vec{J}_{p}(\vec{r}, t)$ are the photo-excited electron and hole current densities, and $n(\vec{r}, t)$ and $p(\vec{r}, t)$ are the photoexcited electron and hole densities, respectively. $\vec{J}_{n}(\vec{r}, t)$ and $\vec{J}_{p}(\vec{r}, t)$ are deduced by subtracting the dark current, provided by the steady-state solution of Eqs. (A12)-(A16) as given later, from the total current within the gap of the PCA. $\mu_{n}$ and $\mu_{p}$ are the electron and hole mobilities, $D_{n}$ and $D_{p}$ are the diffusion coefficients of the electron and hole, and $G(\vec{r}, t)$ and $R(\vec{r}, t)$ are the carrier generation and recombination rates. $\vec{E}_{D C}(\vec{r}), n_{D C}(\vec{r})$, and $p_{D C}(\vec{r})$ are the DC bias field, the electron, and hole densities at the steady-state when the PCA is externally biased. Assuming a Gaussian distribution of the laser beam in both the spatial and temporal domain, the generation rate is determined by

$$
\begin{aligned}
G(\vec{r}, t)= & \frac{\alpha I_{0} \eta}{h \nu} \cdot \exp \left(-\alpha\left(z-z_{0}\right)\right) \\
& \cdot \exp \left(-\frac{\left(x-x_{0}\right)^{2}}{\sigma_{x}^{2}}\right) \cdot \exp \left(-\frac{\left(y-y_{0}\right)^{2}}{\sigma_{y}^{2}}\right) \\
& \cdot \exp \left(-\frac{\left(t-t_{0}-\left(z-z_{0}\right) / v_{\text {semi }}\right)^{2}}{\sigma_{t}^{2}}\right)
\end{aligned}
$$

where $\alpha$ is the absorption coefficient of the semiconductor, $I_{0}$ is the optical peak intensity of the incoming laser pulse, $\eta$ is the optical efficiency due to the reflection at the ambientsemiconductor interface, $\nu$ is the optical frequency, and $h$ is Planck's constant. The coordinate of the modeling domain is shown in Fig. 1. The laser beam propagates in the $z$ direction and the position $\left(x_{0}, y_{0}, z_{0}\right)$ indicates the center point of the laser beam on the top surface of the semiconductor. $t_{0}$ and $\sigma_{t}$ represent the temporal peak and duration of the laser pulse, 
respectively. $\left(\sigma_{x}, \sigma_{y}\right)$ represents the optical beam profile in the cross-section perpendicular to the propagating direction of the laser beam. $v_{\text {semi }}$ is the speed of light in the semiconductor. According to the Shockley-Read-Hall process, the recombination rate is determined by

$$
R(\vec{r}, t)=\frac{n(\vec{r}, t) \cdot p(\vec{r}, t)}{n(\vec{r}, t) \cdot \tau_{p}+p(\vec{r}, t) \cdot \tau_{n}},
$$

where $\tau_{n}$ and $\tau_{p}$ are the electron and hole carrier lifetimes, respectively. The carrier mobilities $\mu_{n}$ and $\mu_{p}$ are fielddependent in reality, which are described as ${ }^{52}$

$$
\begin{gathered}
\mu_{n}=\frac{\mu_{n 0}}{\sqrt{1+\left(\mu_{n 0}\left(\left|\vec{E}+\vec{E}_{D C}\right|\right) / v_{n, s a t}\right)^{2}}}, \\
\mu_{p}=\frac{\mu_{p 0}}{\sqrt{1+\left(\mu_{p 0}\left(\left|\vec{E}+\vec{E}_{D C}\right|\right) / v_{p, s a t}\right)^{2}}},
\end{gathered}
$$

where $\mu_{n 0}$ and $\mu_{p 0}$ are the initial electron and hole mobilities without the external field, and $v_{n, s a t}$ and $v_{p, s a t}$ are the saturated electron and hole velocities, respectively. The carrier mobilities and the diffusion coefficients have the following relation:

$$
\frac{D_{n}}{\mu_{n}}=\frac{D_{p}}{\mu_{p}}=\frac{K_{B} \cdot T}{q},
$$

where $K_{B}$ is Boltzmann's constant, and $T$ is the temperature in Kelvin.

By solving the 3D full-wave model described by Eqs. (A1)-(A6), one can comprehensively simulate the transient behavior of a PCA. To obtain the THz far-field radiation, the near-to-far-field transformation is implemented based on the equivalence principle. $^{42}$

Before starting to solve the full-wave model, the steadystate solution of the DC field $\vec{E}_{D C}(\vec{r})$ and carrier densities $n_{D C}(\vec{r})$ and $p_{D C}(\vec{r})$ of the semiconductor need to be obtained first while the PCA is externally biased. To achieve this, the Poisson equation as well as the carrier dynamics equations should be solved together in the static state, as shown below $^{53-55}$

$$
\begin{gathered}
V_{D C}(\vec{r})=\frac{q}{\varepsilon}\left(n_{D C}(\vec{r})-p_{D C}(\vec{r})-N_{D}+N_{A}\right), \\
\nabla \cdot \vec{J}_{n, D C}(\vec{r})=q R_{D C}(\vec{r}), \\
\nabla \cdot \vec{J}_{p, D C}(\vec{r})=-q R_{D C}(\vec{r}), \\
\vec{J}_{n, D C}(\vec{r})=q \mu_{n} n_{D C}(\vec{r})\left(-\nabla V_{D C}(\vec{r})\right)+q D_{n} \nabla n_{D C}(\vec{r}), \\
\vec{J}_{p, D C}(\vec{r})=q \mu_{p} p_{D C}(\vec{r})\left(-\nabla V_{D C}(\vec{r})\right)-q D_{p} \nabla p_{D C}(\vec{r}),
\end{gathered}
$$

where $V_{D C}(\vec{r})$ is the potential distribution inside the semiconductor, and $N_{D}-N_{A}$ represents the concentration of the impurities. $\vec{J}_{n, D C}(\vec{r}, t)$ and $\vec{J}_{p, D C}(\vec{r}, t)$ are the steady-state current densities of electrons and holes. The recombination rate $R_{D C}(\vec{r})$ is defined as

$$
R_{D C}(\vec{r})=\frac{n_{D C}(\vec{r}) \cdot p_{D C}(\vec{r})-n_{i}^{2}(\vec{r})}{n_{D C}(\vec{r}) \cdot \tau_{p}+p_{D C}(\vec{r}) \cdot \tau_{n}},
$$

where $n_{i}^{2}(\vec{r})$ is the instinct carrier concentration of the semiconductor.

$\vec{E}_{D C}(\vec{r})$ can be easily deduced from $V_{D C}(\vec{r})$ when the steady-state solutions of Eqs. (A12)-(A16) are obtained. Equations (A12)-(A16) are solved using the finite-difference method initially. Once the steady-state solutions are obtained, they are utilized as the initial input parameters of the fullwave model. The 3D finite-difference time-domain (FDTD) method is then applied to obtain the time-domain solution of the full-wave model. The equations are discretized based on Yee's cell, ${ }^{42,56}$ and the numerical implementation of the FDTD algorithm is realized by in-house developed MATLAB codes.

${ }^{1}$ J. Federici and L. Moeller, J. Appl. Phys. 107, 111101 (2010).

${ }^{2}$ P. U. Jepsen, D. G. Cooke, and M. Koch, Laser Photonics Rev. 5, 124 (2011).

${ }^{3}$ D. M. Mittleman, J. Appl. Phys. 122, 230901 (2017).

${ }^{4}$ D. Auston, K. Cheung, and P. Smith, Appl. Phys. Lett. 45, 284 (1984).

${ }^{5}$ P. R. Smith, D. H. Auston, and M. C. Nuss, IEEE J. Quantum Electron. 24, 255 (1988).

${ }^{6}$ Y. Cai, I. Brener, J. Lopata, J. Wynn, L. Pfeiffer, and J. Federici, Appl. Phys. Lett. 71, 2076 (1997).

${ }^{7}$ E. Moreno, M. Pantoja, A. R. Bretones, M. Ruiz-Cabello, and S. G. Garcia, IEEE Antennas Wireless Propag. Lett. 13, 682 (2014).

${ }^{8}$ P. U. Jepsen, R. H. Jacobsen, and S. Keiding, JOSA B 13, 2424 (1996).

${ }^{9}$ Z. Piao, M. Tani, and K. Sakai, Jpn. J. Appl. Phys., Part 1 39, 96 (2000).

${ }^{10}$ Y.-S. Lee, Principles of Terahertz Science and Technology (Springer Science \& Business Media, 2009), Vol. 170.

${ }^{11} \mathrm{X} . \mathrm{-C}$. Zhang and J. Xu, Introduction to THz Wave Photonics (Springer, 2010), Vol. 29.

${ }^{12}$ N. Khiabani, Y. Huang, Y.-C. Shen, S. Boyes, and Q. Xu, "A novel simulation method THz photoconductive antenna characterization," in 2013 7th European Conference on Antennas and Propagation (EuCAP) (IEEE, 2013), p. 751.

${ }^{13}$ N. Khiabani, Y. Huang, Y.-C. Shen, and S. Boyes, IEEE Trans. Antennas Propag. 61, 1538 (2013).

${ }^{14}$ E. Sano and T. Shibata, IEEE J. Quantum Electron. 26, 372 (1990).

${ }^{15}$ P. Kirawanich, S. J. Yakura, and N. E. Islam, IEEE Trans. Plasma Sci. 37, 219 (2009).

${ }^{16}$ E. Moreno, M. F. Pantoja, S. G. Garcia, A. R. Bretones, and R. G. Martin, IEEE Trans. Terahertz Sci. Technol. 4, 490 (2014).

${ }^{17}$ R.-H. Chou, C.-S. Yang, and C.-L. Pan, J. Appl. Phys. 114, 043108 (2013).

${ }^{18}$ E. Moreno, Z. Hemmat, J. Roldán, M. Pantoja, A. Bretones, and S. García, JOSA B 32, 2034 (2015).

${ }^{19}$ S. Chamoun, R. Joshi, E. Arnold, R. Grondin, K. Meyer, M. Pessot, and G. Mourou, J. Appl. Phys. 66, 236 (1989).

${ }^{20} \mathrm{M}$. Van Exter and D. R. Grischkowsky, IEEE Trans. Microwave Theory Tech. 38, 1684 (1990).

${ }^{21}$ M. Tani, S. Matsuura, K. Sakai, and S.-I. Nakashima, Appl. Opt. 36, 7853 (1997).

${ }^{22}$ M. Tani, K. Sakai, and H. Mimura, Jpn. J. Appl. Phys., Part 2 36, L1175 (1997).

${ }^{23}$ F. Miyamaru, Y. Saito, K. Yamamoto, T. Furuya, S. Nishizawa, and M. Tani, Appl. Phys. Lett. 96, 211104 (2010).

${ }^{24}$ N. M. Burford and M. O. El-Shenawee, Opt. Eng. 56, 010901 (2017).

${ }^{25}$ W. Shi, L. Hou, and X. Wang, J. Appl. Phys. 110, 023111 (2011).

${ }^{26}$ X. Ropagnol, M. Bouvier, M. Reid, and T. Ozaki, J. Appl. Phys. 116, 043107 (2014).

${ }^{27}$ E. Castro-Camus, L. Fu, J. Lloyd-Hughes, H. H. Tan, C. Jagadish, and M. B. Johnston, J. Appl. Phys. 104, 053113 (2008).

${ }^{28}$ S.-G. Park, K. H. Jin, M. Yi, J. C. Ye, J. Ahn, and K.-H. Jeong, ACS Nano 6, 2026 (2012).

${ }^{29}$ C. W. Berry, N. Wang, M. R. Hashemi, M. Unlu, and M. Jarrahi, Nat. Commun. 4, 1622 (2013). 
${ }^{30}$ N. Khiabani, Y. Huang, L. E. Garcia-Muñoz, Y.-C. Shen, and A. RiveraLavado, IEEE Trans. Terahertz Sci. Technol. 4, 501 (2014).

${ }^{31}$ S. Lepeshov, A. Gorodetsky, A. Krasnok, E. Rafailov, and P. Belov, Laser Photonics Rev. 11, 177001 (2017).

${ }^{32}$ E. Moreno, R. Sohrabi, G. Klochok, and E. Michael, Optik 166, 257 (2018).

${ }^{33}$ J. T. Darrow, X.-C. Zhang, D. H. Auston, and J. D. Morse, IEEE J. Quantum Electron. 28, 1607 (1992).

${ }^{34}$ A. Taylor, P. Benicewicz, and S. Young, Opt. Lett. 18, 1340 (1993).

${ }^{35}$ J. Holzman, F. Vermeulen, and A. Elezzabi, IEEE J. Quantum Electron. 36, 130 (2000).

${ }^{36}$ G. C. Loata, M. D. Thomson, T. Löffler, and H. G. Roskos, Appl. Phys. Lett. 91, 232506 (2007)

${ }^{37}$ A. Garufo, G. Carluccio, N. Llombart, and A. Neto, IEEE Trans. Antennas Propag. 66, 1635 (2018).

${ }^{38}$ S. E. Ralph and D. Grischkowsky, Appl. Phys. Lett. 59, 1972 (1991).

${ }^{39}$ S. Alexandrou, C.-C. Wang, R. Sobolewski, and T. Y. Hsiang, IEEE J. Quantum Electron. 30, 1332 (1994).

${ }^{40}$ P. C. Upadhya, W. Fan, A. Burnett, J. Cunningham, A. G. Davies, E. H. Linfield, J. Lloyd-Hughes, E. Castro-Camus, M. B. Johnston, and H. Beere, Opt. Lett. 32, 2297 (2007).

${ }^{41}$ E. Moreno, Z. Hemmat, J. Roldán, M. Pantoja, A. Bretones, S. García, and R. Faez, IEEE Trans. Antennas Propag. 64, 4919 (2016).

${ }^{42}$ A. Taflove and S. C. Hagness, Computational Electrodynamics: The Finite-Difference Time-Domain Method (Artech House, 2005).

${ }^{43}$ S.-H. Yang, M. R. Hashemi, C. W. Berry, and M. Jarrahi, IEEE Trans. Terahertz Sci. Technol. 4, 575 (2014)
${ }^{44}$ I. S. Gregory, C. Baker, W. Tribe, M. Evans, H. E. Beere, E. H. Linfield, A. Davies, and M. Missous, Appl. Phys. Lett. 83, 4199 (2003).

${ }^{45}$ D. C. Look, D. Walters, G. Robinson, J. Sizelove, M. Mier, and C. Stutz, J. Appl. Phys. 74, 306 (1993).

${ }^{46}$ D. C. Look, Thin Solid Films 231, 61 (1993).

${ }^{47}$ R. Quay, C. Moglestue, V. Palankovski, and S. Selberherr, Mater. Sci. Semicond. Process. 3, 149 (2000).

${ }^{48}$ D. D. Nolte, J. Appl. Phys. 85, 6259 (1999).

${ }^{49} \mathrm{G}$. Loata, Investigation of Low-Temperature-Grown GaAs Photoconductive Antennae for Continous-Wave and Pulsed Terahertz Generation (UniFrankfurt, 2007).

${ }^{50}$ K. Ezdi, T. Kleine-Ostmann, and M. Koch, "Numerical Simulation of Photoconductive Dipole Antennas: The Effect of the DC Bias Striplines," in 16th International Symposium on Space Terahertz Technology (2005), p. 305.

${ }^{51}$ J. Zhang, M. Tuo, M. Gehl, R. Gibson, M. Liang, G. Khitrova, and H. Xin, Terahertz Emission from Photoconductive Antenna Fabricated on GaAs/ Sapphire Substrate (IEEE, 2015), p. 1.

${ }^{52}$ D. Caughey and R. Thomas, Proc. IEEE 55, 2192 (1967).

${ }^{53}$ D. Vasileska, S. M. Goodnick, and G. Klimeck, Computational Electronics: Semiclassical and Quantum Device Modeling and Simulation (CRC Press, 2017).

${ }^{54}$ W. Fichtner, D. J. Rose, and R. E. Bank, SIAM J. Sci. Stat. Comput. 4, 391 (1983).

${ }^{55}$ J. Zhang, M. Tuo, M. Liang, X. Wang, and H. Xin, Numerical Analysis of Terahertz Generation Characteristics of Photoconductive Antenna (IEEE, 2014), p. 1746.

${ }^{56}$ K. Yee, IEEE Trans. Antennas Propag. 14, 302 (1966). 